\title{
Assessment of Cognitive Impairment in a Mouse Model of High-Fat Diet-Induced Metabolic Stress with Touchscreen-Based Automated Battery System
}

\author{
Saeram Lee ${ }^{1,2 \dagger}$, Jong Youl Kim ${ }^{1 \dagger}$, Eosu Kim ${ }^{2,3}$, KyoungYul Seo ${ }^{4}$, Youn Jae Kang ${ }^{1,2}$, \\ Jae Young Kim ${ }^{1}$, Chul-Hoon Kim ${ }^{2,5}$, Ho Taek Song ${ }^{6}$, Lisa M. Saksida ${ }^{7,8,9}$ \\ and Jong Eun Lee ${ }^{1,2 *}$ \\ ${ }^{1}$ Department of Anatomy, ${ }^{2}$ BK21 PLUS Project for Medical Science, and Brain Research Institute, ${ }^{3}$ Department of Psychiatry, \\ ${ }^{4}$ Department of Ophthalmology and The Institute of Vision Research, ${ }^{5}$ Department of Pharmacology, \\ ${ }^{6}$ Department of Radiology, Yonsei University College of Medicine, Seoul 03722, Korea, ${ }^{7}$ Department of Psychology and \\ MRC/Wellcome Trust Behavioural and Clinical Neuroscience Institute, University of Cambridge, Cambridge, UK, \\ ${ }^{8}$ Canada Research Chair in Translational Cognitive Neuroscience, ${ }^{9}$ Department of Pharmacology \& Physiology, \\ University of Western Ontario, London, ON N6G 2VA, Canada
}

\begin{abstract}
Obesity-related metabolic disorders can affect not only systemic health but also brain function. Recent studies have elucidated that amyloid beta deposition cannot satisfactorily explain the development of Alzheimer's disease (AD) and that dysregulation of glucose metabolism is a critical factor for the sporadic onset of non-genetic $\mathrm{AD}$. Identifying the pathophysiology of $\mathrm{AD}$ due to changes in brain metabolism is crucial; however, it is limited in measuring changes in brain cognitive function due to metabolic changes in animal models. The touchscreen-based automated battery system, which is more accurate and less invasive than conventional behavioral test tools, is used to assess the cognition of mice with dysregulated metabolism. This system was introduced in humans to evaluate cognitive function and was recently back-translated in monkeys and rodents. We used outbred ICR mice fed on highfat diet (HFD) and performed the paired associates learning (PAL) test to detect their visual memory and new learning ability loss as well as to assess memory impairment. The behavioral performance of the HFD mice was weaker than that of normal mice in the training but was not significantly associated with motivation. In the PAL test, the average number of trials completed and proportion of correct touches was significantly lower in HFD mice than in normal diet-fed mice. Our results reveal that HFD-induced metabolic dysregulation has detrimental effects on operant learning according to the percentage of correct responses in PAL. These findings establish that HFD-induced metabolic stress may have an effect in accelerating AD-like pathogenesis.
\end{abstract}

Key words: Metabolic disorders, High-fat diet, Cognitive impairment, Paired Associates Learning test, Alzheimer’s disease

Received July 10,2018, Revised August 3,2018,

Accepted August 6,2018

* To whom correspondence should be addressed.

TEL: 82-2-2228-1646, FAX: 82-2-365-0700

e-mail:jelee@yuhs.ac

"These authors contributed equally to this work.

\section{INTRODUCTION}

Alzheimer's disease $(\mathrm{AD})$, a devastating neurodegenerative
Copyright $\odot$ Experimental Neurobiology 2018. www.enjournal.org
This is an Open Access article distributed under the terms of the Creative Commons Attribution Non-Commercial License (http://creativecommons.org/licenses/by-nc/4.0) which permits unrestricted non-commercial use, distribution, and reproduction in any medium, provided the original work is properly cited. 
condition, leads to progressive cognitive decline and dementia. Classic AD pathology is characterized by extracellular amyloid beta $(A \beta)$ plaques and intraneuronal deposits of neurofibrillary tangles (NFTs). NFTs are constituted by hyperphosphorylated tau proteins, whereas $A \beta$ plaques are insoluble and an aggregated form of $A \beta$ peptide [1]. However, recent studies have focused on the pathology of AD associated with the metabolic syndrome of obesity [2], as clinical trials have failed with respect to drug candidates targeting amyloid pathways, such as beta-secretase inhibitors, gamma-secretase inhibitors, and $A \beta$ itself [3].

The incidence of obesity is steadily increasing worldwide, and $18 \%$ of adults were reported to be obese in 2014 [4]. Obesity is considered one of the greatest threats to human health [5] and is also associated with numerous risk factors, including physical inactivity, genetic components, inflammation, blood lipid disorders, and insulin resistance. These common pathologies are associated with metabolic syndrome, which is a risk factor for neurological diseases $[6,7]$. Therefore, the relationship between obesity and neurodegenerative diseases has been studied with focus on the probability that obesity may result in neurodegeneration, exacerbation of cognitive decline, and increased susceptibility to brain damage $[8,9]$. Recently, obesity has been reported to be associated with not only an increased risk of mild cognitive impairment, but also late-life dementia and AD. The relative risk of dementia and $\mathrm{AD}$ in obese and overweight individuals compared with that in normal weight individuals was 2.04 and 1.64 , respectively [10]. It remains unclear whether obesity alone is related with the risk of AD. Therefore, many researchers have evaluated cognitive function in mouse models through various behavioral tests. However, their studies did not precisely resemble the cognitive tasks used to examine humans. Recent developments in operant chamber technology, such as the touchscreen testing system, have resulted in an increased use of these automated systems with computerized data collection and analysis facilities; in addition to higher translational potential and increased reliability and accuracy, this has also led to the possibility of universal standard protocols across departments in institutions worldwide $[11,12]$. Here, we assessed the performance of ICR mouse subjected to long-term HFD-feeding developing overweight and hyperglycemia, on touchscreen-based fixed ratio (FR) and progressive ratio (PR) schedules and pairedassociates learning (PAL) task, in order to examine motivational changes and cognitive impairment possibly associated with obesity. We also attempted to determine the association between dietinduced obesity and $\mathrm{AD}$ pathologies.

\section{MATERIALS AND METHODS}

\section{Animals}

Total 40 male ICR mice (age: 8 weeks; $\mathrm{n}=20 / \mathrm{ND}$ and 20/HFD; Central Lab Animal Inc., Seoul, Korea) were used in this study and maintained under a 12-h light/dark cycle with food and water ad libitum. One group was provided 60\% high-fat diet (HFD; cat. no. D12492; Research Diets, New Brunswick, NJ, USA), while the other was provided normal diet (ND) with $4.8 \%$ of fat; both groups had free access to food and water. All animal experiments were approved by the Committee for the Care and Use of Laboratory Animals at Yonsei University Health System (2015-0039) and performed in accordance with the National Institute of Health guidelines for the Care and Use of Laboratory Animals.

\section{Body weight and glucose level}

Body weight and fasting blood glucose level were measured every week. Mice were fasted for $4 \mathrm{hr}$ before recording their glucose level. Blood samples were collected by cutting off the tip of the tail, and the glucose level was measured by a glucometer (CareSens II, Meter, Pharmaco Ltd.).

\section{Glucose tolerance test}

The glucose tolerance test is a widely used simple test in clinical practice to diagnose glucose intolerance and type 2 diabetes. Mice were moved to another empty cage for overnight fasting (14 hr). Furthermore, $2 \mathrm{~g} / \mathrm{kg}$ of glucose was dissolved in saline for intraperitoneal injection. Blood was collected from the tip of the tail, and measured with a glucometer at 0,30,60 and 120 min after the injection. The area under the concentration versus time curve (AUC glucose $0 \sim 120 \mathrm{~min}, \mathrm{mg} / \mathrm{dl} \times$ minutes) was calculated and compared.

\section{Insulin tolerance test}

Mice were fasted for $4 \mathrm{hr}$ before the test, and $0.75 \mathrm{U} / \mathrm{kg}$ of insulin was injected intraperitoneally into the subjects (dissolved in saline). Blood was collected from the tip of the tail, and measured with a glucometer at $0,30,60$, and $120 \mathrm{~min}$ after the injection. The area under the concentration versus time curve (AUC glucose 0 120 min, $\mathrm{mg} / \mathrm{dl} \times$ minutes) was calculated and compared.

\section{Touchscreen-automated operant battery system}

Testing was conducted in a Bussey-Saksida touchscreen-based automated operant system for all mice (Campden Instruments Ltd.), as detailed elsewhere $[11,12]$. The apparatus consists of a standard modular testing chamber housed with a sound- and light-attenuating box. The 12-inch-wide touchscreen has a high 
resolution $(800 \times 600)$ and is surrounded by a stainless-steel grid floor and trapezoidal acrylic wall. The inner chamber is surrounded by infrared beams, which detect animal movement. The front beam is located at $6 \mathrm{~cm}$ from the touchscreen, and the rear beam at $3 \mathrm{~cm}$ from the food magazine. Another infrared beam detects head entries to the food magazine. The head entry to the magazine indicates a trial initiation or an attempt to collect a reward. The magazine contains a light-emitting diode (LED), which is turned on when $20 \mu$ of the reward is delivered and is turned off when the mouse collects the reward and exits from the magazine. At the opposite side of the magazine is a flat touchscreen monitor. A black plastic mask (Campden Instruments Ltd) is used to compartmentalize the screen. It has a row of 5 square $(4 \times 4 \mathrm{~cm})$ holes, which are placed $1.5 \mathrm{~cm}$ above the floor. In FR and PR schedules, a visual stimulus only appears in the middle. For the PAL task, masks with 3-square holes were used.

\section{Touchscreen-based behavioral testing}

FR/PR: To determine whether metabolic changes, including overweight, affected the motivation of the animals, FR and PR schedules were conducted prior to the PAL task to evaluate memory. Procedures of FR/PR have been detailed elsewhere [13]. Because the test requires operant learning, which uses strawberry milk as a reward, animals body weights were maintained at $85 \sim 90 \%$ of their average free-fed weight to ensure their motivation for food intake. To acclimate mice to the reward, a small quantity of strawberry milk was provided in their home cage with other food, and the mice were monitored for a full consumption for 3 days to minimize neophobia. For 2 days habituation, mice were introduced to the chamber before the training. For $20 \mathrm{~min}$, mice were exposed to the noise and house light in the chamber and removed thereafter. After the habituation, mice were subjected to the Initial Touch Training. After an intertrial interval (ITI; $5 \mathrm{sec}$ ), a white square was displayed in the center of the windows of the $5 \times 1$ mask. The other grid positions were left blank. After $30 \mathrm{sec}$ of delay, the white image was removed, and reward was delivered with an illumination of the tray light and a 3-KHz tone for $1 \mathrm{sec}$. Entry to collect the reward turns off the tray light and starts the next ITI. If the mice touched the image on the screen while it was displayed, the image was removed, a tone was played with $3 \times$ reward delivered immediately. The completion of 30 trials in 60 min let the mice move on to the FR training. In the FR training, a white square was displayed in the center window of the $5 \times 1$ mask while the other grid positions are left blank. Upon completion of FR5 (five responses make one trial, which provides a single reward) training, animals underwent PR4 task.

PAL: The PAL task was performed as described thoroughly else- where [14]. Briefly, mice first underwent pre-training, which consisted of "initial touch", "must touch","much initiate", and "push blank touch" training. Subsequently, in the PAL training, two of three stimuli appeared on the screen. One stimulus was the correct one $(\mathrm{S}+)$ and another was the incorrect (S-). The other location was left blank without any stimulus. There were 6 possible trial types and 3 stimuli (flower, airplane, and spider). Each stimulus had a designated correct location, and animals were required to learn the paired association between the stimulus and location. A nose poke to the correct $S+$ stimulus resulted in a single bout of reward, that is, $200 \mu$ l of strawberry milk (Seoul Strawberry Milk ${ }^{\circledR}$ ) with magazine light on. A nose poke to the blank window was ignored. After $20 \mathrm{sec}$ of ITI, another trial was initiated. Each session ended when mice completed 36 trials in $60 \mathrm{~min}$, whichever occurred first.

\section{Tissue sample preparation}

After behavior tests, mice were transcardially perfused with saline. Whole brains were dissected at the cortex, hippocampus, and striatum and froze in liquid nitrogen, followed by storage at $-80^{\circ} \mathrm{C}$.

\section{Immunoblotting}

For immunoblotting, the cortex and hippocampus were treated with RIPA lysis buffer (Sigma) consisting of protease inhibitors mixture. Furthermore, $20 \mu \mathrm{g}$ of protein was separated on $10 \%$ sodium dodecyl sulfate-polyacrylamide electrophoresis gels, electrotransferred onto a polyvinylidene difluoride membrane (Milipore), and probed for the protein of interest by incubation with rabbit anti-A $\beta 1-42$ fibril and mouse anti- $\beta$-actin (1:1000; Abcam) at $4^{\circ} \mathrm{C}$ overnight, followed by horseradish peroxidase-conjugated secondary antibodies (1:10000, Abcam) at room temperature for 90 min. Signals were observed using enhanced chemiluminescence reagents (Thermo Fisher Scientific). Images were captured using the LAS 4000 program.

\section{Transmission electron microscopy (TEM)}

Samples was fixed for $12 \mathrm{hr}$ in $2 \%$ glutaraldehyde-paraformaldehyde in $0.1 \mathrm{M}$ phosphate buffer (PB; $\mathrm{pH} 7.4$ ) and washed in $0.1 \mathrm{M}$ phosphate buffer. They were post-fixed with $1 \% \mathrm{OsO}_{4}$ dissolved in $0.1 \mathrm{M}$ PB for $2 \mathrm{hr}$ and dehydrated in an ascending gradual series (50 100\%) of ethanol and infiltrated with propylene oxide. Specimens were embedded using Poly/Bed 812 kit (Polysciences). After pure fresh resin embedding and polymerization at $65^{\circ} \mathrm{C}$, samples were put in the electron microscope oven (TD-700, DOSAKA) for $24 \mathrm{hr}$. Approximately 200-25-nm-thick sections were initially cut and stained with toluidine blue (Sigma, T3260) and observed under a light microscope. Furthermore, 70-nm-thin sections were double-stained with 6\% uranyl acetate (EMS, \#22400) for $20 \mathrm{~min}$ 
and lead citrate (Fisher, for $10 \mathrm{~min}$ ) for contrast staining. The sections were cut by LEICA EM UC-7 (Leica Microsystems) with a diamond knife (Diatome) and transferred on copper and nickel grids. All thin sections were observed through transmission electron microscopy (JEM-1011, JEOL) at an acceleration voltage of $80 \mathrm{kV}$.

A Formvar-carbon coated EM grid was placed on top of the brain tissue drop for approximately $1 \mathrm{~min}$. The grid was removed, blotted with a filter paper, and placed onto a drop of $2 \%$ uranyl acetate for $15 \mathrm{~s}$. The excess uranyl acetate was removed, and the EM grid was examined and photographed using transmission electron microscopy (JEM-1011, JEOL).

\section{Hematoxylin and eosin staining}

The eye specimen was fixed with 4\% paraformaldehyde and embedded with paraffin. Excised sections were roughly 5 - $\mu \mathrm{m}$ thick. Sections were stained with hematoxylin and eosin (H\&E; Muto, Japan).

\section{Tonometry}

Mice were anesthetized with an intraperitoneal injection of xylazine $\left(10 \mathrm{mg} / \mathrm{kg}\right.$; Rompun ${ }^{\circledR}$, Bayer Animal Health) and zolazepam and tiletamine (30 mg/kg; Zoletil 50). Intraocular pressure (IOP) was measured using a rebound tonometer (Icare ${ }^{\circledR}$ TONOLAB tonometer, Colonial Medical Supply). IOP measurements were recorded according to the manufacturer's instructions. One trial result was given after six consecutive measurements, and the mean of 10 consecutive trials was considered for analyses. Mouse phenotyping analysis was performed by Korea Mouse Phenotyping Center.

\section{Optical coherence tomography}

After anesthesia, the pupils were dilated with $0.5 \%$ tropicamide and $0.5 \%$ phenylephrine-mixed eye drop (Mydrin-P, Santen Pharmaceutical Co, Ltd.). . Optical coherence tomography (OCT) scans were obtained using Micron ${ }^{\circledR}$ IV (Phoenix Research Labs, Pleasanton, CA, USA). The cornea was lubricated with hypromellose 2.5\% (Goniovisc, Hub Pharmaceuticals), and mice were placed collaterally to the OCT camera on the right side of a platform fixed in front of the OCT lens. Subsequently, we focused on the retina and obtained fundus photography and OCT scans. Retinal thickness was measured using InSight-Animal OCT Segmentation Software (Phoenix Research Labs).

\section{Electroretinogram}

Electroretinogram (ERG) recordings were obtained using Micron Ganzfeld ERG (Phoenix Research Labs, Pleasanton). Mice were dark-adapted overnight for at least $12 \mathrm{hr}$ before the experiment for scotopic testing (rod cell response). After anesthesia, the pupils were dilated as previously described. Once the pupil was adequately dilated, we applied hypromellose 2.5\% (Goniovisc) and inserted the electrodes. ERG was recorded as Ganzfeld ERG according to the standard protocol of the manual instruments. Scotopic ERGs were obtained in response to increasing flash intensities, ranging from $-1.7 \log \mathrm{cd} \cdot \mathrm{s} / \mathrm{m}^{2}$ to $1.9 \log \mathrm{cd} \cdot \mathrm{s} / \mathrm{m}^{2}$. Photopic ERGs were obtained in response to increasing flash intensities, ranging from $-0.5 \log \mathrm{cd} \cdot \mathrm{s} / \mathrm{m}^{2}$ to $4.1 \log \mathrm{cd} \cdot \mathrm{s} / \mathrm{m}^{2}$. Ten responses to light stimulation were averaged. The a-wave (as a measure of photoreceptor function), b-wave (as a measure of bipolar cell function), and amplitude and implicit times of the rod and cone responses were determined.

\section{Statistical analyses}

All studies were randomized and assessments were performed by investigators blinded to the experimental conditions. All data were analyzed with standard statistical methods (t-test; Systat Software, Inc). Data are presented as the mean \pm standard error. Differences between the two groups were compared using an unpaired t-test, and multiple comparisons were performed using one-way ANO$\mathrm{VA}$, followed by Bonferronis post hoctest. $\mathrm{p} \leq 0.05$ was considered significant.

\section{RESULTS}

\section{Long-term HFD feeding increased body weight and blood glucose level}

Long-term HFD feeding resulted in a 1.5-fold higher body weight relative to ND (Fig. 1A). Blood glucose levels significantly increased in HFD mice compared with ND mice (Fig. 1B). However, independent of the elevated blood glucose level, HFD mice had no significant effect in either glucose tolerance or insulin resistance (Fig. 1C 1F).

\section{Metabolic dysregulation had no effect on the motivation level in HFD mice}

In many neurodegenerative diseases and psychiatric disorders including $\mathrm{AD}$, reduced motivation is observed. To assess motivation level and reward-related decision making, we used FR and PR paradigms [13]. Eight-week-old mice were randomly assigned to two groups and fed either a normal or high-fat diet for 12 weeks. After 2 weeks of weight regulation by chow deprivation, fixed and progressive ratio tests were conducted. At 27 weeks of feeding, mice started the PAL test and proceeded until 42 weeks before they were sacrificed. To learn the FR task for poking mice's heads into 
A

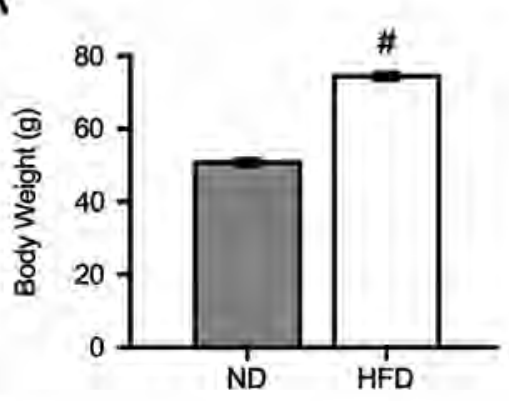

C

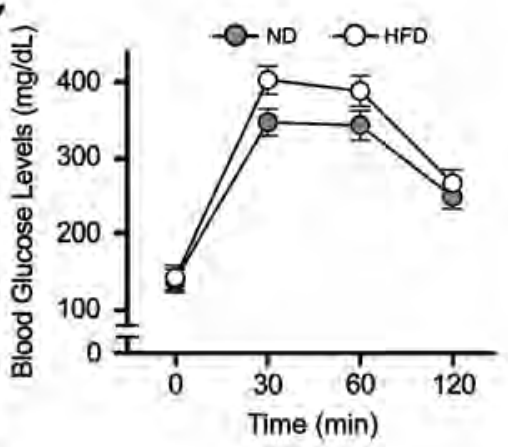

E

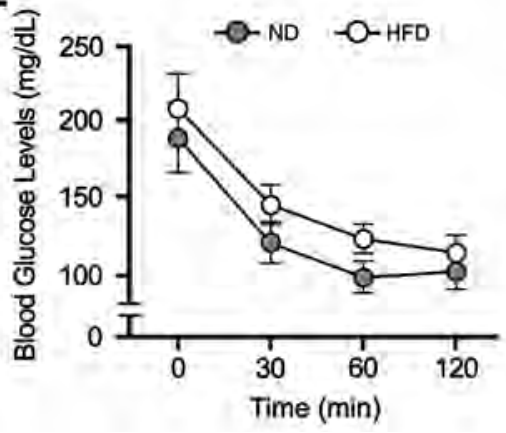

B

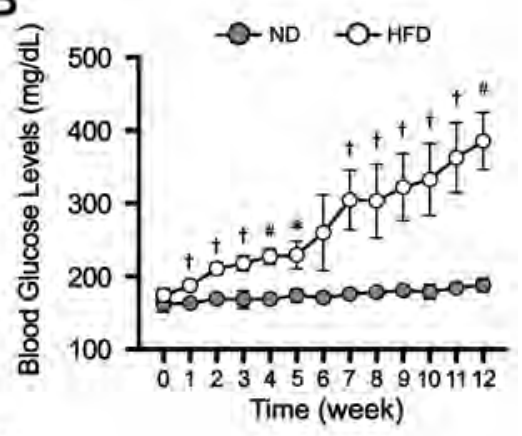

D

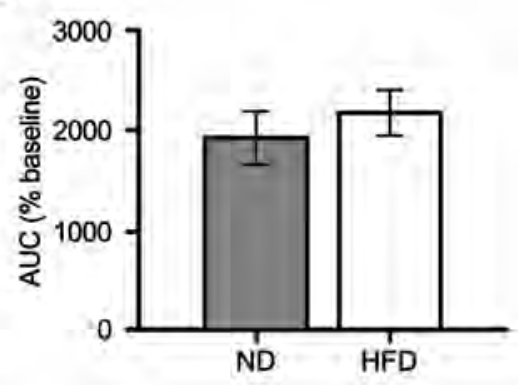

F

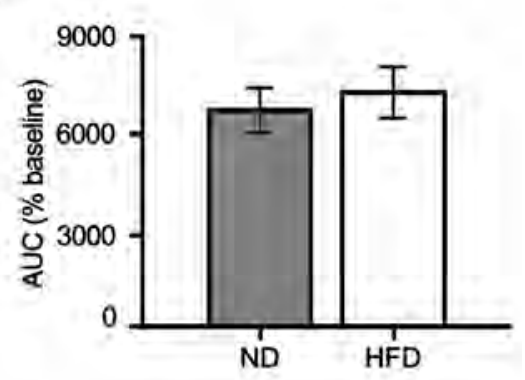

Fig. 1. Body weight and blood glucose level of ND and HFD mice. (A) Effect of HFD on body weight. Male ICR mice were fed an HFD supplement for 12 weeks. (B) Weekly blood glucose levels of ND and HFD mice measured from 8 to 20 weeks of age. (C) Blood glucose levels during the intraperitoneal glucose tolerance test (IPGTT). (D) The area under the curve of the glucose during the IPGTT. (E) Blood glucose levels during the intraperitoneal insulin tolerance test (IPITT). (F) The area under the curve of the glucose during the IPITT $\left(n=18 / \mathrm{ND}\right.$ and $17 / \mathrm{HFD}$; ${ }^{*} \mathrm{p}<0.05$, $\left.{ }^{\dagger} \mathrm{p}<0.005,{ }^{\sharp} \mathrm{p}<0.001\right)$. the food magazine, the body weight of feeding-controlled ND and HFD (ND and HFD ctrl) mice was reduced (about 10 15\%) compared with the average body weight (a.w.) of 12-week-old feedingfree mice (Fig. 2B). Mice were first trained in FR1 (single touch in a trial to provide a single reward) and moved on to FR3, and to FR5 (five correct touches in a trial). Sessions to Criteria, which indicates the number of sessions (days) required to achieve the criterion of each step, significantly differed between the groups; the HFD group needed more time to complete the training conducted from FR1 to FR5 (Fig. 2C). Upon completion of FR5 training, mice were transferred to PR4, and the behavioral performances in PR4 were measured by breakpoint, which indicates the number of responses in the last completed ratio condition. The average breakpoint of three sessions did not differ between the groups (Fig. 2D). The data indicate that motivation was not significantly affected by HFD, although instrumental (operant) learning in the FR schedule was slower in HFD mice than in ND mice.

\section{Metabolic dysregulation induced learning impairment in HFD mice}

To assess the cognitive changes affected by the HFD, we used the PAL paradigm. In the pre-training phase of the PAL task, HFD mice showed a slower progress than did the ND mice (Fig. 3A). In the PAL task, mice were trained to learn associations between the object (shape) and location. Despite 14 sessions of PAL training, most animals from both ND and HFD groups hardly completed all of the 36 trials in $60 \mathrm{~min}$. We thought this reduced level of task engagement might be because of their age, high body weight, or perceptual (visual) disturbance. Thus, instead of continuing the training, we decided to use the last three sessions to present results of the PAL performance of these animals (Fig. 3B D). As shown by the average session length, most animals did not complete the 
A

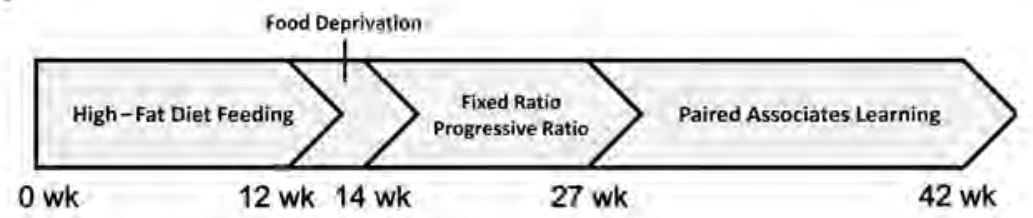

B

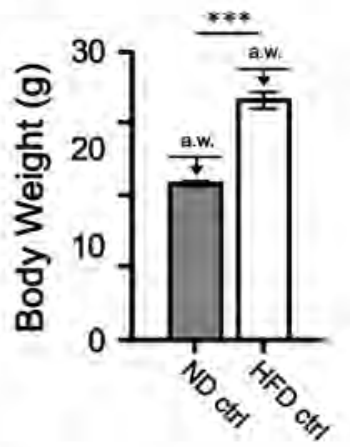

D

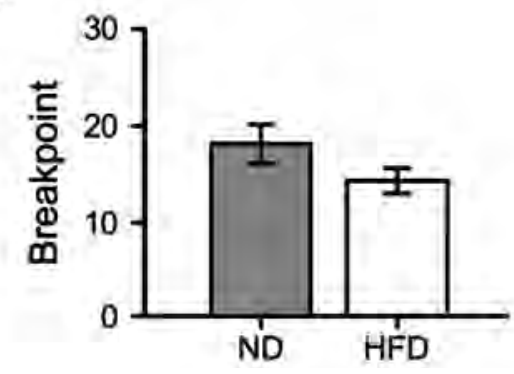

C

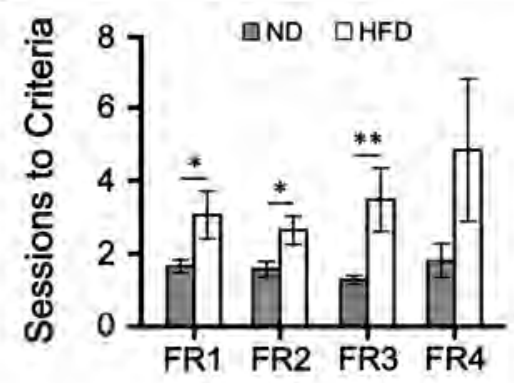

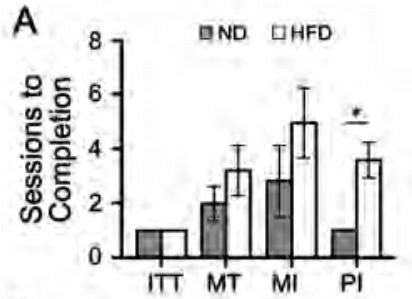

C

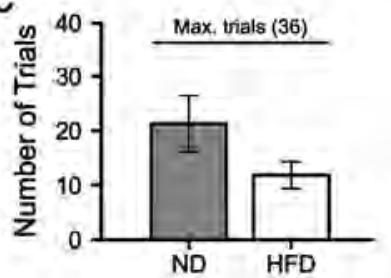

B

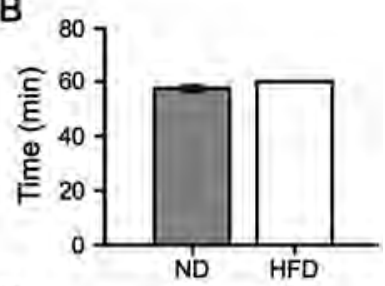

D

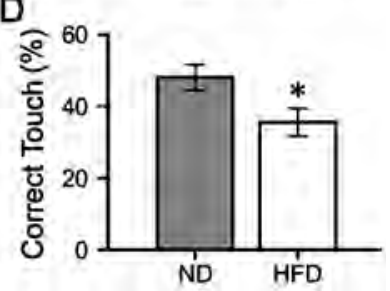

Fig. 3. Rate of training and response accuracy of ND and HFD mice in Paired-Associates Learning (PAL) schedules. (A) Performance of male ICR mice in PAL training for 60 minutes. (B) Average session length in the last 3 sessions of PAL task (Sessions ended in $60 \mathrm{~min}$ ). (C) The average number of trials per session conducted in the last three sessions of the PAL task. (D) Accuracy in the last three sessions of the PAL task ( $n=7 / N D$ and $11 /$ HFD group; ${ }^{*} \mathrm{p}<0.05$ ).

Fig. 2. Performance of feeding-controlled ND and HFD mice in the progressive ratio 4 (PR4) schedule. (A) Timeline of the behavioral study. (B) Body weight of ND and HFD mice after the food restriction. (C) The number of sessions that met criteria for the fixed ratio (FR) training. (D) The mean PR4 breakpoint of three consecutive sessions of test $\left(\mathrm{n}=13 / \mathrm{ND}\right.$ and $16 /$ HFD group; $\left.{ }^{*} \mathrm{p}<0.05,{ }^{* *}<0.005\right)$.

36 trials within $60 \mathrm{~min}$ in these three sessions (Fig. 3B). The average number of trials completed was significantly lower for HFD mice (Fig. 3C). The percentage of correct touch (accuracy=correct responses divided by correct plus incorrect responses, \%) was also significantly lower in HFD mice compared with ND mice, suggesting HFD-induced learning impairment (Fig. 3D).

\section{HFD led to decreased number of synapses and elevated $A \beta$ fibril expression in mice}

To explore structural changes in hippocampal neurons of HFD mice, we used electron microscopy and immunoblotting. The number of synapse in the hippocampus was significantly decreased and post-synaptic densities were thinned in HFD mice fed for 24 weeks (Fig. 4A). In the hippocampal synapses of ND mice, $56 \pm 3 \%, 48.5 \pm 5 \%$, and $38 \pm 4 \%$ were preserved in the CA1, CA 3 , and DG, respectively. HFD feeding significantly reduced the numbers of synapses in the CA1, CA3, and DG of hippocampus by $38 \pm 2 \%$, $27 \pm 1.5 \%$, and $25 \pm 2 \%$ (Fig. $4 \mathrm{~B}$ ). To confirm the expression of $\mathrm{A} \beta$ 
A
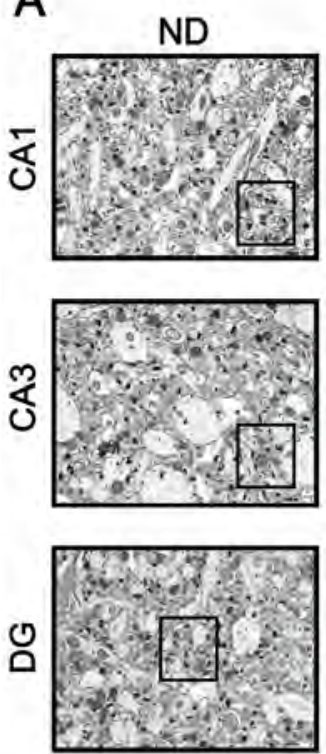

High Mag
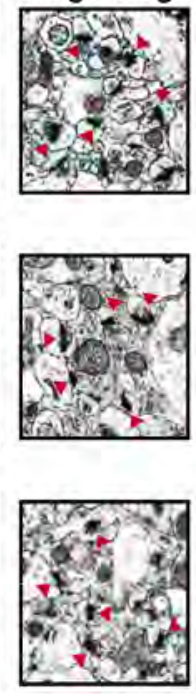
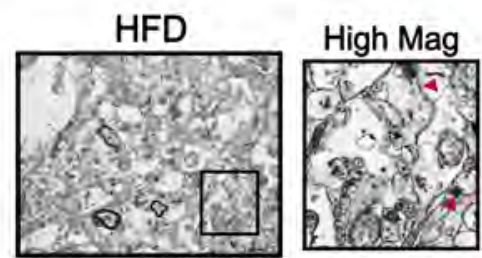
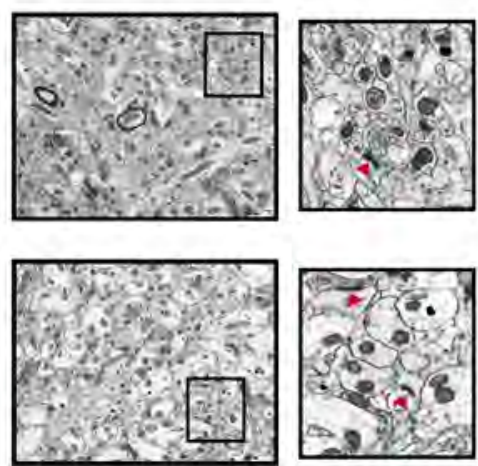

B

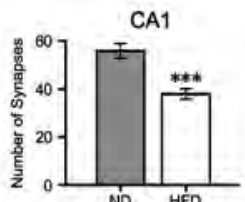

DG
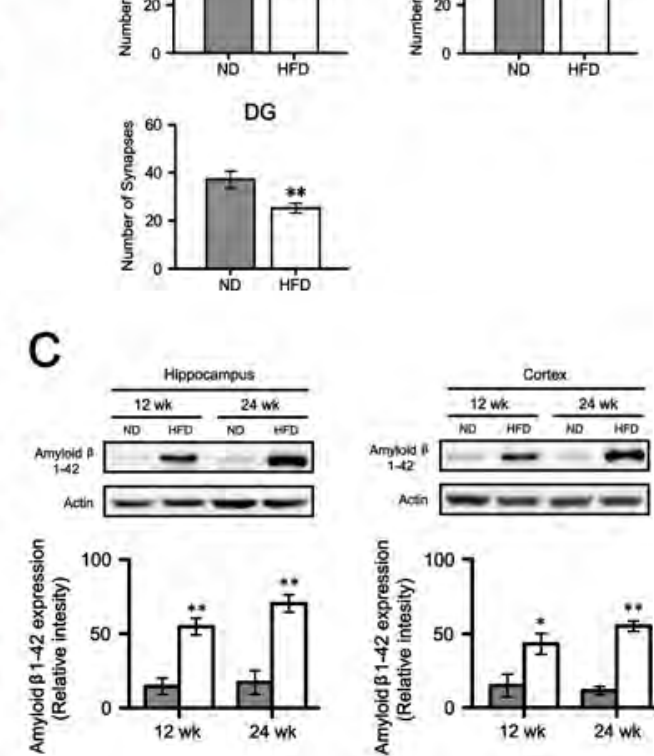

Fig. 4. Synaptic morphology changes and A $\beta$ fibril expression in ND and HFD mice. (A) TEM images of CA1, CA3, and DG in the hippocampus. In the high magnification (High Mag), the red triangles are pointing to the post synaptic density. (B) The numbers of synapse in TEM images. (C) A $\beta$ fibril expression of the hippocampus and cortex in the ND and HFD groups at 12 and 24 weeks. Relative intensities of A $\beta$ fibrils to $\beta$-actin in the hippocampus and cortex ( $\mathrm{n}=3 / \mathrm{ND}$ group and $3 / \mathrm{HFD}$ group; $\left.{ }^{*} \mathrm{p}<0.05,{ }^{* *} \mathrm{p}<0.01,{ }^{* * *} \mathrm{p}<0.001\right)$.

after long-term HFD feeding in the mice brain, immunoblots were also performed. The expression of $A \beta$ fibrils were increased in the cortex and hippocampus of HFD mice on 12 and 24 weeks (Fig. $4 \mathrm{C}$ ). These data support that the metabolic dysregulation by longterm HFD feeding induces neuronal injury.

\section{Long-term HFD mice had no change in visual acuity}

Using touchscreen-automated battery system with visual stimulation, visual function was a critical issue. Visual tests were conducted to confirm that visual acuity was not affected by dietinduced diabetes-like symptom, therefore, behavioral test result is solely based on the cognitive decline [15]. Tonometry data suggest no significant difference in intraocular pressure between ND and HFD mice, suggesting no chances for glaucoma (Fig. 5A). MannWhitney tests showed no morphological difference either in the cornea or fundus between ND and HFD mice. The layer of their retina was also well-distinguished and showed the same ratio of IPL:INL:ONL-RPE (Fig. 5B). The morphological structure of the H\&E-stained sections showed slight, but not significant swelling (Fig. 5C). In the retina of ND and HFD mice, the function of the rod and cone cell had no significant effect (Fig. 5D). Analysis of the A- and B-wave amplitude indicated that function and reaction latencies had no significant difference in photoreceptor cells of
ND and HFD mice. Therefore, in ND and HFD mice, structures and functions of the cornea and retina were intact, and the function of photoreceptor cells also presented a normal state (Fig. 5E).

\section{DISCUSSION}

Our study demonstrates cognitive function failure induced by metabolic dysregulation in a rodent model. In the HFD group, the blood glucose level increased along with body weight gain, in a time-dependent manner; this group also showed difficulty in learning simple tasks. In support of the learning disability, the number synapse was reduced in the CA1, CA3, and DG of the hippocampus of HFD mice. On the other hand, $A \beta$ expression in the cortex and hippocampus of HFD mice was increased compared with that in the ND mice.

The global prevalence of obesity is increasing. Obesity is an excessive accumulation of fat stored in adipose and non-adipose tissues as triglycerides that can be broken down into fatty acids, which can negatively affect health [16]. Obesity is an important factor for the development of metabolic disorders [17]; it has damaging effects on many organ systems. Many complications and concurrent diseases are related to metabolic syndrome, characterized by a high weight, glucose intolerance, high triglyceride levels, 
A

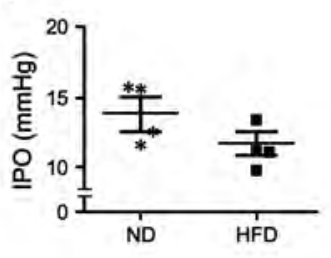

D

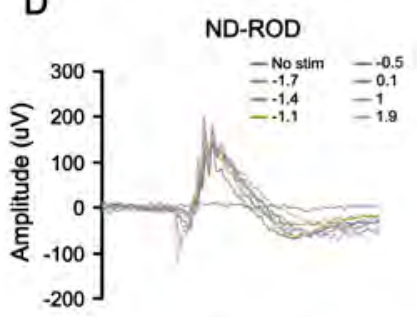

ND-CONE

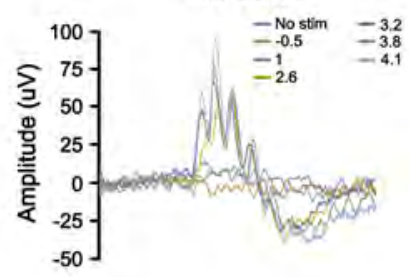

B
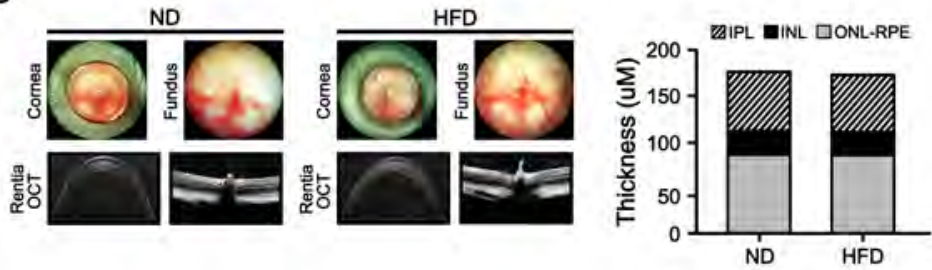

$E$
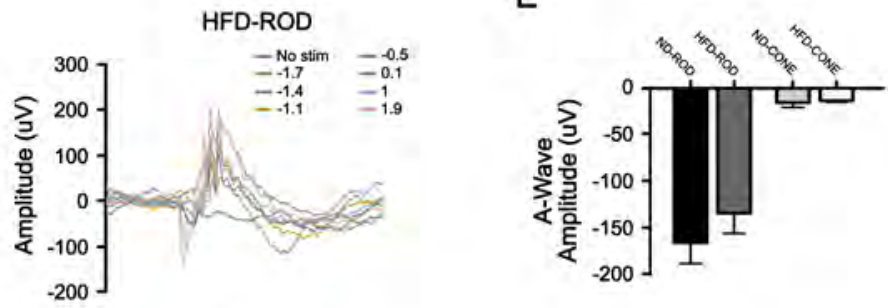

HFD-CONE

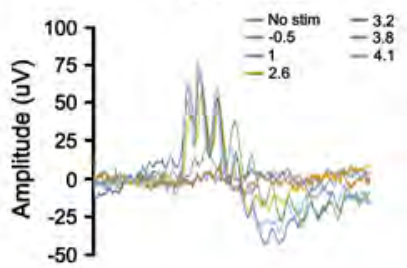

C

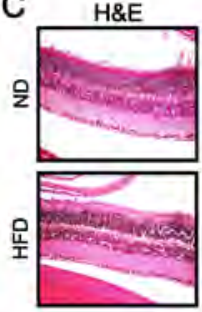

Fig. 5. Visual test of ND and HFD mice. (A) Intraocular pressure of the ND and HFD groups. (B) The corneal and retinal morphology of the ND and HFD groups, and layer distribution of the retina measured on optical coherence tomography. (C) H\&E staining of the retina. (D) Electroretinography of the ND and HFD groups showing light sensitivity of the rod cells, cone cells, and connecting ganglion cells of each mouse. (E) The quantification of the light sensitivity of the retina to the A- and B-wave and their response time ( $\mathrm{n}=4 / \mathrm{ND}$ group and $4 / \mathrm{HFD}$ group).

and hypertension and are thus risk factors for non-insulin-dependent (type II) diabetes mellitus (T2DM), coronary artery diseases, systemic hypertension, and heart failure. Moreover, obesity is associated with the incidence of respiratory diseases such as obstructive sleep apnea, gastrointestinal and musculoskeletal disorders, thromboembolism, stroke, and cancer [18, 19]. In the past decade, it has been widely accepted that HFD leads to obesity that causes chronic low-grade inflammation followed by insulin resistance [17]. Subsequently, insulin resistance leads to hyperinsulinemia and $\beta$-cell failure successively, resulting in various metabolic disorders, including type II diabetes and hepatic steatosis. However, in our study, HFD mice did not develop either glucose tolerance or insulin resistance, suggesting a prediabetic state than T2DM [20]. In this study, we investigated non-diabetic metabolic disease. Recently, obesity induced by metabolic syndrome is reported to be concurrent with $\mathrm{AD}$, indicating that $\mathrm{AD}$ is a type of metabolic disease [21]. Therefore, metabolic alteration could be the potential cause of $\mathrm{AD}$, such that various substances (insulin, adiponectin, and antioxidants) influenced by metabolic alterations could be a therapeutic target for $\mathrm{AD}$.
Recent epidemiological and clinical studies have indicated that $\mathrm{A} \beta$ deposition cannot satisfactorily explain $\mathrm{AD}$ development, and that dysregulation of glucose metabolism is a critical factor for the sporadic onset of the non-genetic, vast majority form of $\mathrm{AD}$ [22]. The development of $\mathrm{AD}$ treatment could benefit from considering the relationship between $\mathrm{AD}$ and obesity. Over the last decade, the population of obese people has increased in epidemic proportions. Therefore, the association between obesity and neurodegenerative diseases has been studied with focus on the probability that obesity may lead to neurodegeneration, exacerbation of cognitive decline, and increased susceptibility to brain damage $[8,9]$. In this regard, numerous studies have shown that people with midlife obesity (measured by body mass index or central adiposity) have an augmented risk of AD [23]. According to previous studies, HFD increases hippocampal oxidative stress, which reduces NF-E2related factor 2 signaling [24], causes mitochondrial homeostasis deficiency [25], and impairs hippocampus-dependent memory function [26]. Therefore, measuring the impairment of cognitive function due to a HFD in an animal model, especially the hippocampus-dependent memory function, is crucial. The development 
of robust and validated cognitive assays in mice is essential to fully capitalize on this species, which is increasingly used in neurobiological investigations and as models of diseases involving the hippocampus, such as schizophrenia and $\mathrm{AD}[27,28]$. However, the method of cognitive behavioral testing in animal models remains limited, mostly for measuring spatial cognitive ability. Trustworthy and reproducible analysis of animal learning and behavior is an important factor for basic and translational neuroscience research $[29,30]$. Current advancement inoperant chamber technology, such as the touchscreen testing system, has resulted in the expending use of these automated systems with computerized data accumulation and analysis facilities. Furthermore, higher translational potential and increased reliability and accuracy have contributed to the possibility of universal standard protocols across departments in institutions worldwide $[11,12,31]$. Thus, hippocampusdependent memory function changes caused by HFD were assessed using touchscreen methods in this study. Behavioral testing of the animals using the touchscreen method showed that longterm HFD did not affect motivation levels for feeding. This study shows that metabolic control disorders caused by HFDs do not affect the level of motivation for experimental animal feeding. In the touchscreen behavioral test, HFD animals showed slow progress in the PAL training session. As the PAL training progressed slowly, long-term HFD was observed to influence the memory acquisition of mice. These results reveal that metabolic control disorder caused by HFD causes learning disorder. However, the animals used in this experiment were very old (age: 50 weeks) and were fed high fat for a long time at the completion of the behavioral test. In fact, animals were hardly engaged in the PAL task, as indicated by the incomplete sessions (Fig. 3B and C). This might be because of their old age, high body weights, or perceptual disturbances especially in visual ability. In this sense, despite collateral evidence on abnormalities in hippocampal neurons, our data from PAL tasks only indicate a nonspecific failure in operant learning itself rather than any particular form of learning (such as associates learning) in HFD mice as their PAL performance was even lower than the chance level (50\%; Fig. 3D), in addition to their general difficulty observed in pre-training (Fig. 2C and 4A).

In order to rule out visual perception loss in HFD mice, the retina and visual function were examined. No structural or cellular difference was observed in the visual system of ND and HFD mice. The retina of HFD mice was slightly swollen, but the structure and functions of the cornea and retina were intact; photoreceptor cell function was also normal. Therefore, in this study, we investigated the cognitive functions of various areas of experimental animals in addition to spatial cognitive ability through behavioral screening using touchscreen methods.
Next, we investigated the expression of $A \beta$ fibrils and changes in hippocampal neurons at the synapses in order to relate this deterioration of cognitive function caused by high-fat feeding to structural changes in neurodegenerative diseases such as AD. In our study, the number of axons was reduced and the expression of $A \beta$ was increased in hippocampal neurons of the HFD mice. Long-term high fat intake also led to a loss of synapses. At 24 weeks in the HFD group, the number of synapse per square area was significantly reduced; in particular, post-synaptic density was significantly reduced. These results suggest that this structural degradation may lead to a decrease in cognitive function.

Our results demonstrate that metabolic control disorders induced by HFD result in an impairment in cognitive function in animals, and that this cognitive dysfunction is caused by the structural loss of the hippocampal neurons. These results suggest that HFD-induced metabolic stress may have an effect in accelerating pathogenesis of AD-like cognitive function.

\section{ACKNOWLEDGEMENTS}

This study was supported by a grant from the Korea Health Technology R\&D Project through the Korea Health Industry Development Institute, funded by the Ministry of Health \& Welfare, Republic of Korea (HI14C2173) to JEL. The authors would like to thank Hae-sol Shin and HongKyung Kim in Korea Mouse Phenotyping Center for their assistance with mouse phenotyping analysis.

\section{REFERENCES}

1. Mucke L (2009) Neuroscience: Alzheimer's disease. Nature 461:895-897.

2. Tolppanen AM, Ngandu T, Kåreholt I, Laatikainen T, Rusanen M, Soininen H, Kivipelto M (2014) Midlife and late-life body mass index and late-life dementia: results from a prospective population-based cohort. J Alzheimers Dis 38:201-209.

3. Cummings JL, Morstorf T, Zhong K (2014) Alzheimer's disease drug-development pipeline: few candidates, frequent failures. Alzheimers Res Ther 6:37.

4. Nguyen JC, Killcross AS, Jenkins TA (2014) Obesity and cognitive decline: role of inflammation and vascular changes. Front Neurosci 8:375.

5. Caveney E, Caveney BJ, Somaratne R, Turner JR, Gourgiotis L (2011) Pharmaceutical interventions for obesity: a public health perspective. Diabetes Obes Metab 13:490-497.

6. Aitlhadj L, Avila DS, Benedetto A, Aschner M, Stürzenbaum SR (2011) Environmental exposure, obesity, and Parkinson's 
disease: lessons from fat and old worms. Environ Health Perspect 119:20-28.

7. Mardinoglu A, Kampf C, Asplund A, Fagerberg L, Hallström BM, Edlund K, Blüher M, Pontén F, Uhlen M, Nielsen J (2014) Defining the human adipose tissue proteome to reveal metabolic alterations in obesity. J Proteome Res 13:5106-5119.

8. Ashrafian H, Harling L, Darzi A, Athanasiou T (2013) Neurodegenerative disease and obesity: what is the role of weight loss and bariatric interventions? Metab Brain Dis 28:341-353.

9. Wolozin B (2004) Cholesterol and the biology of Alzheimer's disease. Neuron 41:7-10.

10. Anstey KJ, Cherbuin N, Budge M, Young J (2011) Body mass index in midlife and late-life as a risk factor for dementia: a meta-analysis of prospective studies. Obes Rev 12:e426-e437.

11. Horner AE, Heath CJ, Hvoslef-Eide M, Kent BA, Kim CH, Nilsson SR, Alsiö J, Oomen CA, Holmes A, Saksida LM, Bussey TJ (2013) The touchscreen operant platform for testing learning and memory in rats and mice. Nat Protoc 8:1961-1984.

12. Mar AC, Horner AE, Nilsson SR, Alsiö J, Kent BA, Kim CH, Holmes A, Saksida LM, Bussey TJ (2013) The touchscreen operant platform for assessing executive function in rats and mice. Nat Protoc 8:1985-2005.

13. Heath CJ, Bussey TJ, Saksida LM (2015) Motivational assessment of mice using the touchscreen operant testing system: effects of dopaminergic drugs. Psychopharmacology (Berl) 232:4043-4057.

14. Bartko SJ, Vendrell I, Saksida LM, Bussey TJ (2011) A computer-automated touchscreen paired-associates learning (PAL) task for mice: impairments following administration of scopolamine or dicyclomine and improvements following donepezil. Psychopharmacology (Berl) 214:537-548.

15. Gong CY, Lu B, Hu QW, Ji LL (2013) Streptozotocin induced diabetic retinopathy in rat and the expression of vascular endothelial growth factor and its receptor. Int J Ophthalmol 6:573-577.

16. Chen WW, Zhang X, Huang WJ (2016) Role of neuroinflammation in neurodegenerative diseases (Review). Mol Med Rep 13:3391-3396.

17. Osborn O, Olefsky JM (2012) The cellular and signaling networks linking the immune system and metabolism in disease. Nat Med 18:363-374.

18. Grundy SM (2004) Obesity, metabolic syndrome, and cardiovascular disease. J Clin Endocrinol Metab 89:2595-2600.

19. Haslam DW, James WP (2005) Obesity. Lancet 366:11971209.
20. Sima AA, Shafrir E (2003) Animal models in diabetes: a primer. Harwood Academic Publishers, Amsterdam.

21. Kang S, Lee YH, Lee JE (2017) Metabolism-centric overview of the pathogenesis of Alzheimer's disease. Yonsei Med J 58:479-488.

22. Song MK, Bischoff DS, Song AM, Uyemura K, Yamaguchi DT (2017) Metabolic relationship between diabetes and Alzheimer's disease affected by Cyclo(His-Pro) plus zinc treatment. BBA Clin 7:41-54.

23. Profenno LA, Porsteinsson AP, Faraone SV (2010) Metaanalysis of Alzheimer's disease risk with obesity, diabetes, and related disorders. Biol Psychiatry 67:505-512.

24. Morrison CD, Pistell PJ, Ingram DK, Johnson WD, Liu Y, Fernandez-Kim SO, White CL, Purpera MN, Uranga RM, Bruce-Keller AJ, Keller JN (2010) High fat diet increases hippocampal oxidative stress and cognitive impairment in aged mice: implications for decreased Nrf2 signaling. J Neurochem 114:1581-1589.

25. Petrov D, Pedrós I, Artiach G, Sureda FX, Barroso E, Pallàs M, Casadesús G, Beas-Zarate C, Carro E, Ferrer I, Vazquez-Carrera M, Folch J, Camins A (2015) High-fat diet-induced deregulation of hippocampal insulin signaling and mitochondrial homeostasis deficiences contribute to Alzheimer disease pathology in rodents. Biochim Biophys Acta 1852:1687-1699.

26. McNeilly AD, Williamson R, Sutherland C, Balfour DJ, Stewart CA (2011) High fat feeding promotes simultaneous decline in insulin sensitivity and cognitive performance in a delayed matching and non-matching to position task. Behav Brain Res 217:134-141.

27. Papaleo F, Lipska BK, Weinberger DR (2012) Mouse models of genetic effects on cognition: relevance to schizophrenia. Neuropharmacology 62:1204-1220.

28. Hall AM, Roberson ED (2012) Mouse models of Alzheimer's disease. Brain Res Bull 88:3-12.

29. Crabbe JC, Wahlsten D, Dudek BC (1999) Genetics of mouse behavior: interactions with laboratory environment. Science 284:1670-1672.

30. Steckler T (2015) Preclinical data reproducibility for R\&D-the challenge for neuroscience. Psychopharmacology (Berl) 232:317-320.

31. Nithianantharajah J, Komiyama NH, McKechanie A, Johnstone M, Blackwood DH, St Clair D, Emes RD, van de Lagemaat LN, Saksida LM, Bussey TJ, Grant SG (2013) Synaptic scaffold evolution generated components of vertebrate cognitive complexity. Nat Neurosci 16:16-24. 